Rev. Elev. Méd. vét. Pays trop., 1972, 25 (3) : 357-366

\title{
Entérite épizootique des bovins à Madagascar
}

\author{
par H. SERRES $\left(^{*}\right)$ et J. BLANCOU $\left(^{*}\right)$
}

\begin{abstract}
RESUME
Une entérite épizootique sévit en hiver austral chez les zébus de Madaga_car. Escherichia coli joue un rôle important dans l'évolution de la maladie, sans que son rôle étiologique soit exclusivement démontré. La thérapeutique antibactérienne et une vaccination anti-colibacillaire suffisent à maîtricer l'épizootie.
\end{abstract}

En hiver austral, des cas d'entérite diarrhéique sont souvent observés chez le zébu malgache (8). Mais jusqu'en 1955, cette entérite n'avait jamais été considérée comme une entité morbide bien définie et, à ce titre, ne retenait pas l'attention des vétérinaires.

Ce n'est qu'à partir de l'année 1955 qu'elle s'est répandue de façon spectaculaire et a causé le plus de pertes parmi la population bovine, justifiant des recherches spéciales sur la maladie, son étiologie et sa prophylaxie.

Certaines de ces recherches se sont poursuivies depuis et ce sont leurs résultats et leurs conclusions que résume cette note.

\section{DESCRIPTION CLINIQUE DE LA MALADIE}

\section{Symptômes}

L'entérite atteint essentiellement les bovins adultes, sans distinction de sexe. épargnant généralement les jeunes animaux. Elle se présente sous deux formes distinctes : aiguë ou chronique.

\section{Entérite aiguë}

Le malade est atteint brutalement, sans prodrome marqué, d'une diarrhée profuse.

(*) I.E.M.V.T., Région de Recherches Vétérinaires et Zootechniques de Madagascar, B.P. $n^{\circ} 862$, Tananarive.
Dans certains cas, seule une courte période d'inappétence et de constipation est notée.

Les émissions de fèces sont très fréquentes, liquides, projetées en jets pouvant atteindre plusieurs mètres. Par certains aspects, cette diarrhée peut rappeler celle que provoque la coccidiose, bien que la présence de sang (sous forme de légères traînées) soit très exceptionnelle.

Les matières fécales sont brunes, parfois verdâtres ou jaunâtres, d'odeur butyreuse. Il est net que cette diarrhée provient d'un intestin enflammé, car elle est chaude et "fume longtemps» comme le disent les paysans, au contraire de l'entérite paratuberculeuse. Dès l'apparition de la diarrhée, l'animal cesse de s'alimenter et paraît abattu. Sa soif est intense. La température rectale reste toujours voisine de $39^{\circ}$, sauf dans certains cas où une courte phase fébrile $\left(40^{\circ}\right)$ peut être mise en évidence. Le malade maigrit alors assez rapidement et, si aucune amélioration ne survient spontanément, meurt en 8 à 10 jours environ.

Par certains aspects, cette entérite rappelle celle observée chez les bovins adultes d'autres pays tels que le Canada, l'Australie, l'Angleterre ou les Etats-Unis, sous le nom de "Winter dysentery " $(2,4,5,6,7,10,11)$.

\section{Entérite chronique}

Les bovins atteints d'entérite aiguë ne suc- 
combent pas toujours. Fréquemment la diarrhée s'amende progressivement et devient chronique : les excréments se raffermissent. Les guérisons, si elles ne paraissent pas rares, sont toujours lentes. L'animal recouvre un appétit irrégulier durant un mois environ, est amaigri et présente une muqueuse pâle ou sub-ictérique. Il reste affaibli, facilement victime d'une affection intercurrente, d'autant plus que l'éleveur l'isole le plus souvent loin du troupeau et sans abri contre les intempéries.

\section{Lésions}

Nous distinguerons les lésions visibles à l'autopsie de celles décelables seulement par examen histologique.

\section{Lésions macroscopiques}

Sur le cadavre frais, on peut observer:

Dans les cas d'entérite aiguë. - Une inflammation généralisée de toute la muqueuse digestive, particulièrement nette au niveau de la caillette dont la sous-muqueuse est presque toujours hémorragique. Ces lésions sont parfois accompagnées d'une péritonite aiguë, localisée, à larges placards hémorragiques et fibrineux, avec une tuméfaction des plaques de PEYER. Tous les autres viscères sont normaux, sauf quelques pétéchies parfois constatées au niveau du cœur et de l'aorte.

Dans le cas d'entérite chronique. - Sur le cadavre en état de cachexie avancée, les lésions d'entérite dominent, mais la caillette est moins enflammée que dans la forme aiguë.

\section{Lésions microscopiques}

Deux organes essentiellement présentent des lésions histologiques constantes: la caillette, siège de lésions inflammatoires aiguës avec accumulation de polynucléaires entre muqueuse et sous-muqueuse et l'intestin grêle, siège de lésions inflammatoires aiguës et subaiguës accompagnées d'importante infiltration polynucléaire de la sous-muqueuse, entre les villosités intestinales.

\section{Epizootiologie}

\section{Régions atteintes}

Les régions de Madagascar où sont observés le plus fréquemment des cas d'entérite sont celles des Hauts Plateaux (Antsirabe, Itasy, Betafo) et celles des régions montagneuses du Nord (Bealanana) et de l'Est (Lac Alaotra), régions considérées par ailleurs comme les plus froides de l'Ile. Dans ces régions, la maladie apparaît essentiellement comme une affection de bas-fonds, Ies localités les plus éprouvées s'inscrivant généralement dans un périmètre marécageux. Les troupeaux les plus atteints sont ceux qui pâturent en zones basses et les ęleveurs, qui ont observé le fait, les déplacent sur les hauteurs dès les premiers cas de maladie. Les épizooties surviennent généralement dans une zone orographique bien déterminée, suggérant une origine hydrique possible du contage.

\section{Périodes d'épizootie}

La maladie survient le plus souvent durant la saison froide qui se situe, à Madagascar, entre le mois de juin et le mois d'octobre. Un relevé des températures minimales durant cette période, dans une des régions les plus touchées (plateau de l'Ankaizina, au Nord de l'Ile) indique l'ordre de grandeur des températures locales. Il faut noter que cet abaissement thermique est souvent aggravé par une hygrométrie élevée, pouvant atteindre 87 p. 100 lors des matinées les plus froides:

TABLEAU $\mathrm{N}^{*} \mathrm{I}$

\begin{tabular}{|c|c|c|c|c|c|c|c|c|c|c|c|c|}
\hline Annểe Mois & Janv. & Fёv. & Mars & Avm. & Mai & Juin & Juil. & Août & Sept. & Oct. & Nov. & Déc. \\
\hline 1958 & $16^{\circ} 9$ & $17^{\circ} 3$ & $16^{\circ} 6$ & $15^{\circ} 1$ & $12^{\circ} 5$ & $11^{\circ} 9$ & $10^{\circ} 2$ & $10^{\circ} 6$ & $10^{\circ} \mathrm{g}$ & $15^{\circ} 9$ & $14^{\circ} 2$ & $16^{\circ} 2$ \\
\hline 1959 & $16^{\circ} 1$ & $16^{\circ} 9$ & $17^{\circ} 6$ & $15^{\circ} \mathrm{I}$ & $11 * 6$ & $10^{\circ} 4$ & $9^{\circ} 6$ & $10^{\circ}$ & $9 \% 4$ & $10^{\circ} 9$ & $15^{\circ} 4$ & $16^{\circ}$ \\
\hline 1960 & $16^{\circ} 4$ & $15^{\circ} 6$ & $16^{\circ} 5$ & $14^{\circ} 6$ & $13^{\circ} 7$ & $12^{\circ} 2$ & $10^{\circ}$ & $10^{\circ} 5$ & $B^{\circ} B$ & $10^{\circ} 9$ & $12^{\circ} 4$ & $16^{\circ}$ \\
\hline $\begin{array}{l}\text { Nombre de foyers } \\
\text { rapportês en } \\
10 \text { ans }\end{array}$ & 2 & 1 & 0 & 0 & 1 & 1 & 1 & 4 & 6 & 10 & 3 & 2 \\
\hline
\end{tabular}




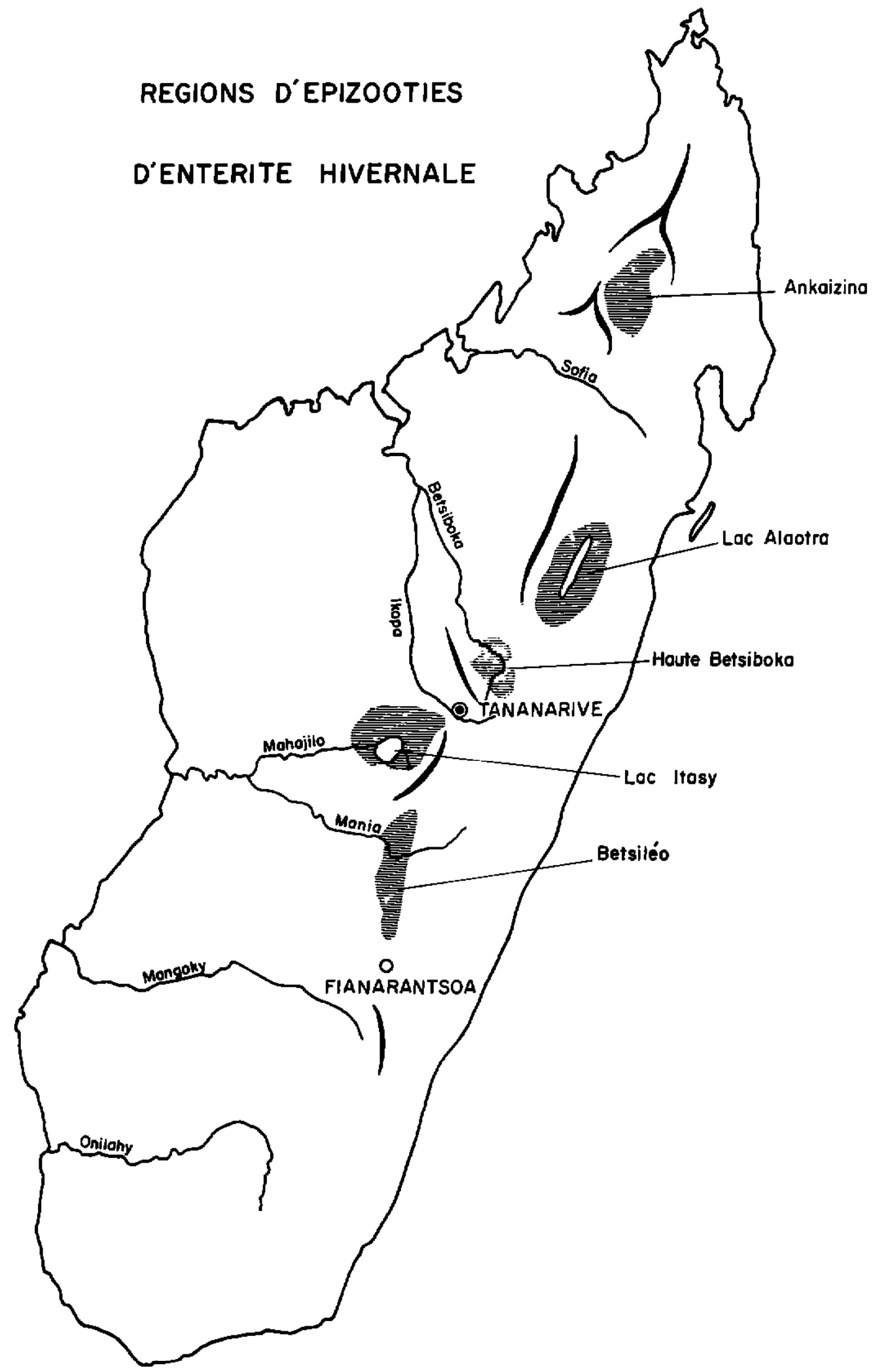


Dans ce tableau, on peut constater la relation qui existe entre la saison et la fréquence des épizooties. Des foyers d'entérite, généralement bénigne, peuvent survenir en-dehors de la saison la plus froide comme cela fut le cas récemment en 1970-1971. Il est à souligner que plusieurs expériences, entre autres d'alimentation du bétail, nous ont montré que le zébu malgache, qui provient essentiellement des savanes côtières de l'Ouest, est très sensible au froid dès qu'on le déplace vers les régions de consommation, en altitude.

\section{Evolution de l'épizootie}

La maladie évolue par vagues successives, certains foyers étant plus étendus et plus persistants que d'autres. La morbidité et la mortalité restent relativement faibles. Elles peuvent être illustrées par les chiffres suivants rapportés par F. NICOLAS (8) lors de l'épizootie survenue en 1957 au Lac Alaotra:

«Sur une région comptant 133.000 bovins recensés, 7.511 furent atteints d'entérite $(15,6 \mathrm{p}$. $100)$ et 1.520 en moururent (1,14 p. 100$)$.

Dans certaines régions, plus touchées, la mortalité atteignait 3,5 p. 100 de l'effectif. "

\section{RECHERCHE SUR L'ETIOLOGIE DE LA MALADIE}

Toutes les causes possibles de la maladie ont été systématiquement recherchées. Les méthodes utilisées dans ce but, et les résultats obtenus concernant les divers agents possibles de l'entérite sont les suivants :

\section{Protozoaires}

\section{Méthodes de recherche}

Des frottis de sang colorés au May-Grunwald-Giemsa et des prélèvements d'excréments ont été exécutés sur les lieux même de l'épizootie et examinés au microscope.

\section{Résultats}

Aucun hématozoaire pathogène n'a jamais été décelé, quel que soit le stade de la maladie. Aucune coccidie n'a jamais été observée dans les fèces.

\section{Helminthes}

\section{Méthodes de recherche}

Coproscopie après enrichissement ou recherche directe des helminthes dans l'intestin des cadavres autopsiés.

\section{Résultats}

Les helminthes les plus souvent rencontrés étaient, par ordre de fréquence Carmyerius dollfusi, Paramphistomum cervi, Botriophoron Botriophoron et Cooperia sp. Cependant ces parasites n'étaient jamais observés en nombre suffisant pour expliquer les troubles mortels constatés. Par ailleurs, des sujets témoins, sains, présentaient un parasitisme identique. Il faut noter que la fasciolose à Fasciola gigantica (8) a pu interférer, à partir de 1969, avec l'entérite épizootique mais sans jamais en être la cause directe.

\section{Virus}

\section{Méthodes de recherche}

Trois méthodes ont été mises en cuvre:

Inoculation aux bovins. - Des fèces diarrhéiques, diluées au 1/10 en eau distillée puis filtrées sur Seitz $E K S$ 2, ont été inoculées à dix bovins par voie parentérale selon la méthode décrite par L.W. MacPHERSON (7) pour isoler le virus de la "Winter dysentery".

Inoculation en culture cellulaire. - Les mêmes fèces, additionnées d'antibiotiques, ont été inoculées sur cellules rénales de veau.

Recherche d'anticorps contre la maladie des muqueuses. - Le pouvoir neutralisant en culture cellulaire de 65 sérums de bovins de régions touchées par l'entérite a été éprouvé vis-à-vis de la souche $\mathrm{MI}$ de la maladie des muqueuses (8).

\section{Résultats}

Aucun pouvoir pathogène pour le bovin ni pour les cultures cellulaires n'a pu être décelé après les inoculations (8).

Aucun sérum de bovins ne présentait d'anticorps neutralisant par la souche MI de la maladie des muqueuses. Ces deux résultats, ajoutés au fait que l'antibiothérapie s'est toujours montrée régulièrement efficace dans le traitement de l'entérite, ne plaident pas en faveur d'une origine virale, sans bien sûr l'exclure définitivement. 


\section{Toxiques}

Aucune recherche systématique de toxique n'a pu être entreprise. Mais, outre que les caractères épizootiologiques de l'affection ne sont pas en faveur d'une étiologie toxique, l'examen attentif de la nourriture des malades comparée à celle des témoins a toujours permis de l'écarter.

La consommation de manioc vert, parfois suspectée, n'entraîne aucun trouble chez les animaux des troupeaux voisins.

\section{Bactéries}

C'est finalement l'étiologie qui paraissait la plus vraisemblable compte tenu de tous les caractères cliniques et épizootiologiques de l'entérite, et du succès constant de l'antibiothérapie.
Toutes les espèces bactériennes susceptibles de provoquer un syndrome entérique ont été recherchées par bactérioscopie, mise en culture sur milieux solides et liquides (enrichis ou sélectifs) des prélèvements suivants: sang, moelle osseuse, rate, ganglions mésentériques, foie, fèces.

Les genres Shigella, Pasteurella, Vibrio, Spirocheta, Mycobacterium n'ont jamais été isolés. Les genres Staphylococcus, Welchia, Salmonella étaient parfois présents dans les fèces mais toujours en quantité insuffisante pour provoquer des troubles intestinaux. $\mathrm{La}$ seule espèce bactérienne retrouvée en quantité anormale dans les fèces ou présente dans les organes des cadavres était Escherichia coli. Sur 325 prélèvements, cette bactérie a été isolée 224 fois selon le détail suivant:

TABLEAU $\mathrm{N}^{\circ} \mathrm{II}$

\begin{tabular}{|c|c|c|c|}
\hline \multirow{2}{*}{ Origine des prélèvements } & \multicolumn{3}{|c|}{$\begin{array}{c}\text { Isolements réussis à partir } \\
\text { des prëlèvements ci-dess ous }\end{array}$} \\
\cline { 2 - 4 } & F è c e s & 0 g 1 o n g s \\
\hline Lac Alaotra & 52 & 11 & 10 \\
\hline Arkaızına & 8 & 10 \\
\hline Autres lieux & 72 & 32 & 9 \\
\hline
\end{tabular}

Il faut souligner que de nombreux isolements, en culture pure d'emblée, ont été obtenus à partir de la moelle osseuse, ce qui démontre une septicémie colibacillaire.

Par ailleurs, le sérum des bovins malades agglutine les souches isolées de leur organisme jusqu'à la dilution $1 / 160$, ce qui ne se produit jamais chez les bovins sains.

Quinze parmi les souches isolées des cas les plus graves ont été adressées, pour sérotypie, à Copenhague (Docteur F. ØRSKOV) et à l'Institut Pasteur de Paris (Docteur A. VALLEE). Elles appartenaient aux sérotypes suivants :

02H8 - 01/0149H8 - 0106/0113H21 $027 / 068-064-08-06-0110-079-$ 034/0140 - 082 - 08 - 024/010/014 - H19 H8.

On notera l'hétérogénéité de ces divers sérotypes. Escherichia coli, paraissant donc la seule espèce bactérienne constamment suspecte, a fait l'objet de recherches particulières quant à son rôle dans la pathogénie de l'entérite.

\section{RECHERCHES \\ SUR LE ROLE PATHOGENIQUE D'ESCHERICHIA COLI}

Dans ces recherches, nous distinguons le pouvoir pathogène des souches à l'isolement de celui des souches ayant subi plusieurs repiquages successifs.

\section{Pouvoir pathogène à l'isolement}

Sur 45 souches étudiées, 30 se sont révélées mortelles pour la souris, à la dose de 0,025 à $0,05 \mathrm{ml}$ de culture en bouillon (inoculée par voie sous-cutanée). Par contre, même à l'isolement, ces souches sont sans action sur les bovins quelle que soit la voie d'inoculation (doses de 1 à $50 \mathrm{ml}$ ). 


\section{Pouvoir pathogène expérimental}

Nous désignons par ce terme le pouvoir pathogène recherché avec des souches repiquées. Nous exposons les résultats obtenus avec la souche $\mathrm{O} 2 \mathrm{H} 8$ (qui a subi déjà plus de 200 repiquages) mais les résultats sont les mêmes avec d'autres souches.

Le pouvoir pathogène de cette souche est considérablement affaibli comme l'indiquent les effets d'une culture en bouillon sur les animaux suivants :

Cobayes, souris : ni l'abreuvement quotidien durant dix jours, ni l'inoculation intra-péritonéale de $0,1 \mathrm{ml}$ de culture n'entraînent de troubles chez ces deux espèces.

Bovins: sur des zébus, adultes ou jeunes, l'ingestion de $1.000 \mathrm{ml}$ de la culture tous les 3 jours (pendant 15 jours) non plus que l'inoculation intra-péritonéale de $500 \mathrm{ml}$ ou intraveineuse de $10 \mathrm{ml}$ n'entraînent de trouble. Seule l'ingestion quotidienne de $500 \mathrm{ml}$ de culture provoque, au $6^{\mathrm{e}}$ jour, un syndrome diarrhéique transitoire, non fébrile. Mais l'ingestion de fèces de malades (200 à $500 \mathrm{~g}$ ) ne provoque aucun trouble.

Afin d'exalter ce pouvoir pathogène, nous avons utilisé les artifices qui nous paraissaient le mieux reproduire les conditions naturelles auxquelles sont soumis les animaux en période d'épizootie, à savoir :

- le froid, et son action dépressive sur l'organisme;

- la sécheresse et ses conséquences : concentration des points d'eau (avec modification de ces caractères chimiques et bactériologiques) et sous-alimentation des bovins (avec affaiblissement général et modification de la flore intestinale).

Les résultats sont les suivants :

1. Pouvoir pathogène d'Escherichia coli pour des animaux déprimés par le froid:

Souris: la dose léthale 50 p. 100 sur deux lots de 30 souris, l'un laissé à température ambiante $\left(16-24^{\circ}\right)$, l'autre placé à $+4^{\circ}$, est de $0,4 \mathrm{ml}$ contre $0,1 \mathrm{ml}$ (par voie intrapéritonéale).

Cobayes: la dose léthale 50 p. 100 sur deux lots de 20 cobayes inoculés par voie intra-péritonéale est de $3 \mathrm{ml}$ si l'inoculum est à $37^{\circ}$ contre $1 \mathrm{ml}$ s'il est à $16^{\circ}$.

2. Pouvoir pathogène d'Escherichia coli pour des animaux déprimés par une sousalimentation :

Six zébus malgaches ont été soumis à une diète de 15 jours (alimentation réduite à un peu de paille sèche). Ils ont alors reçu, ainsi que six zébus témoins normalement nourris, $500 \mathrm{ml}$ de culture de colibacille per os chaque jour. Ces animaux à jeun ont été atteints de diarrhée plus précocement que les témoins ( $2^{\mathrm{e}}$ jour) mais aucun n'a été gravement malade.

3. Pouvoir pathogène d'Escherichia coli pour des animaux à flore intestinale déséquilibrée :

Cobayes: un lot de 20 cobayes est prétraité aux antibiotiques (Pénicilline 1.000 UI et Streptomycine $0,1 \mathrm{cg}$ ) puis éprouvé avec 20 témoins par $1 \mathrm{ml}$ de culture. Aucun trouble chez les témoins, 12 malades et 6 morts chez les cobayes sous antibiotiques.

Bovins: nous avons tenté de modifier l'équilibre de leur flore intestinale par modification du $\mathrm{pH}$ de l'eau de boisson. Cette eau, en saison sèche, acquiert une acidité marquée (pH 5,5 à 5) susceptible de modifier la réceptivité des bovins à l'infection. Sur cinq bovins ayant reçu per os de la culture colibacillaire ramenée à $\mathrm{pH} \mathrm{5,} \mathrm{trois} \mathrm{ont} \mathrm{réagi} \mathrm{par} \mathrm{une}$ poussée thermique $\left(40^{\circ}\right)$ et une diarrhée marquée, mais aucun des animaux n'est mort.

Les trois types d'expériences précédentes suggèrent une influence possible de ces trois facteurs associés: froid, sous-alimentation, déséquilibre de la flore intestinale. Ils ne suffisent cependant pas à entraîner la mort des bovins, ni à reproduire le tableau complet de la maladie naturelle. Peut-être faudrait-il faire appel à la notion physiopathologique nouvelle d'hypersensibilisation par la lyse des bactéries, signalée en matière d'entérite du porc par plusieurs auteurs $(1,12,13)$ ou à celle de la coagulation intravasculaire disséminée (3), " phénomène déclenché par une micropopulation non spécifique qui entraînerait l'organisme dans un cercle vicieux conduisant à la mort, tout en favorisant l'extension de l'affection dans l'élevage". 
Ces deux notions ont l'avantage de rendre compte du succès de l'antibiothérapie ou de la vaccination sur des entérobactéries habituellement saprophytes.

\section{TRAITEMENT DE L'ENTERITE}

\section{Etude de laboratoire}

La responsabilité d'Escherichia coli étant prépondérante dans l'entérite épizootique, la sensibilité de 55 souches, isolées de cas graves, a été recherchée in vitro par la méthode des disques ou des tubes. Toutes les souches éprouvées sont très sensibles à la Furazolidone, à la Streptomycine, à la Néomycine, au formol. Elles sont irrégulièrement sensibles à la Tífomycine (36 souches sur 41), la Terramycine (6 sur 31), la Sulfadimérazine (13 sur 55), au Ganidan (34 sur 55), au Novarsénobenzol (10 sur 15$)$.

\section{Essai sur le terrain}

Au cours des nombreuses épizooties, divers traitements ont été institués, tous avec succès : Alunozal (50 à $100 \mathrm{~g}$ par jour), Ganidan (30 à $50 \mathrm{~g}$ par jour), Novarsénobenzol ( $3 \mathrm{~g}$ par voie intraveineuse), Formol (20 ml de formol du commerce en solution à 5 p. 100 par voie intraveineuse).

Antibiotiques: Pénicilline, Streptomycine, Terramycine aux doses habituelles.

Cependant, il n'a pas toujours été aisé de contrôler l'efficacité réelle de chacun de ces traitements car ils étaient souvent institués simultanément. Toutefois les observations de F. NICOLAS (8) concernant des animaux traités par le Novarsénobenzol ou le formol donnent une idée statistique de l'efficacité du traitement.

Nombre de malades non traités: 1.122; morts : 929, soit 82 p. 100.

Nombre de malades traités : 6.449 ; morts : 591, soit 9 p. 100 .

\section{PROPHYLAXIE}

Les mesures de prophylaxie sanitaire classiques : isolement des malades, destruction des cadavres, enfouissement des litières, se sont toujours montrées efficaces lorsqu'elles étaient applicables. Mais dans la pratique courante. et compte tenu des habitudes des éleveurs, elles l'étaient rarement.

Dès l'apparition des premières épizooties, c'est donc plutôt vers une prophylaxie médicale que se sont portés les espoirs des vétérinaires traitants.

Un vaccin a été préparé à partir d'une culture en milieu liquide de deux souches très pathogènes à l'isolement (de sérotype 02H8 et H19). La culture est neutralisée par addition de 5 p. 1000 de formol. Elle titre $10^{8}$ bactéries par ml. Le vaccin est inoculé à la dose de $2 \mathrm{ml}$ par voie sous-cutanée.

La valeur de ce vaccin a été reconnue sur le terrain et contrôlée en laboratoire.

\section{Valeur du vaccin sur le terrain}

Elle peut être illustrée par le tableau (p. 364) établi en 1957 à la suite d'une enquête détaillée sur les résultats des mesures de prophylaxie instituées.

On peut citer l'exemple de l'exploitation M... au Lac Alaotra qui possédait environ 500 bovins. La vaccination fut pratiquée sur 320 d'entre eux, la quantité de vaccin disponible n'étant pas suffisante pour la totalité du troupeau. Quinze jours plus tard, la maladie faisait son apparition, entraînant 18 cas dont six mortels, uniquement dans l'effectif non vacciné.

\section{Contrôle du vaccin en laboratoire}

Compte tenu de l'absence de pouvoir pathogène expérimental des souches de colibacille pour le bovin, ce contrôle doit être exécuté par sérologie in vitro ou épreuve sur souris.

In vitro. - L'évolution du taux des anticorps chez les bovins vaccinés a été suivie par la méthode de l'hémagglutination passive (8) trois semaines après la vaccination.

Avant vaccination, le sérum des animaux ne 


\begin{tabular}{|c|c|c|c|c|}
\hline $\begin{array}{c}\text { Mesures } \\
\text { instituêes }\end{array}$ & $\begin{array}{c}\text { Nombre } \\
\text { de sujets }\end{array}$ & $\begin{array}{l}\text { Nombre de malades } \\
1 \text { à } 10 \text { jours après }\end{array}$ & $\begin{array}{l}\text { Nombre de malades } \\
10 \text { a } 20 \text { jours après }\end{array}$ & $\begin{array}{c}\text { Nombre de malades } \\
\text { aprẽs } 20 \text { jours }\end{array}$ \\
\hline $\begin{array}{l}\text { Vaccination en } \\
\text { milieu indemne }\end{array}$ & 15.293 & réant & néant & néant \\
\hline $\begin{array}{l}\text { Vaccination en } \\
\text { milieu infecté } \\
\text { (maladie } \\
\text { déclarée) }\end{array}$ & 7.258 & 88 & 10 & néant \\
\hline $\begin{array}{l}\text { Vaccination en } \\
\text { milieu infecte } \\
\text { (r.aladie en } \\
\text { incubation) }\end{array}$ & 2.063 & 43 & 25 & néant \\
\hline $\begin{array}{l}\text { Absence de } \\
\text { vaccination }\end{array}$ & 26.000 & 5.000 & - & - \\
\hline
\end{tabular}

provoque pas d'hémagglutination des hématies sensibilisées au-delà d'une dilution au 1/20. Après vaccination, sur 50 sérums de bovins, 5 avaient conservé le même taux d'anticorps, 20 atteignaient le taux de $1 / 40,22$ celui de $1 / 80$ et 3 celui de $1 / 160$.

Sur souris. - Sur 80 souris témoins éprouvées par $0,5 \mathrm{ml}$ de culture (voie intra-péritonéale) : 70 mortes. Sur 80 souris vaccinées 13 jours auparavant avec $0,5 \mathrm{ml}$ de vaccin " bovin »: 17 mortes.

L'antigénicité et l'immunogénicité du vaccin semblent donc réelles, tant au laboratoire qu'en pratique. Le service vétérinaire de Madagascar utilise actuellement plus de deux millions de doses de ce vaccin chaque année.

\section{CONCLUSION}

L'entérite épizootique qui sévit chez le zébu malgache avec une gravité variable selon les années et les régions, paraît, dans l'état actuel des recherches, être d'origine bactérienne, mais non transmissible, et liée à des facteurs favorisants.

Sans que la démonstration absolue ait pu en être faite, car elle nécessiterait l'emploi d'un grand nombre de bovins soumis à de nombreux stress difficilement reproductibles en laboratoire, la bactérie Escherichia coli joue sûrement un rôle déterminant dans la maladie et les mortalités qu'elle entraîne. L'intervention primitive d'un autre agent (viral?) n'est pas exclue, mais non démontrée.

Les résultats obtenus par une thérapeutique et une prophylaxie médicale dirigées uniquement contre Escherichia coli donnent actuellement toute satisfaction dans la pratique.

\section{SUMMARY}

\section{Bovine epizootic enteritis in Madagascar}

An epizootic enteritis occurs in malagasy zebu cattle during the austral winter season.

Escherichia coli plays furst in the disease, but its aetiological part may be not exclusive.

Antibiotic therapy and colibacillosis vaccine are able to control the disease.

\section{RESUMEN}

Enteritis epizootica de los bovinos en Madagascar

Una enteritis epizootica ocurre en los cebus de Madagascar durante el invierno austral. Escherichia coli desempeña un papel importante en la 
evolución de la enfermedad, sin que se demuestre exclusivamente su papel etiologico.

La terapeutica antibacteriana y una vacunación anticolibacilar son suficientes para suprimir la epizootia.

\section{BIBLIOGRAPHIE}

1. BUXTON (A.) et THOMLIWSON (J.R.). The detection of tissue-sensitizing antibodies to Escherichia coli in oedema disease, haemorrhagic gastro-enteritis and in normal pigs. Res. vet. Sci., 1961, $2: 78-88$.

2. EDWARDS (M.J.) et SIER (A. M.). Bovine epizootic diarrhoea in Western Australia Aust. vet. J., 1960, $36: 402-404$.

3. ESPINASSE (J.). Dimetridazole et typhlo-colite nécro-hémorragique. Essai d'interprétation. Cah. méd. vét., 1971, 40 (3): 95-99.

4. HEDSTROM (H.) et ISAKSSON (A.). Epizootic enteritis in cattle in Sweden. Cornell Vet., 1951, 4 : 251-253.

5. JONES (F. S.) et LITTLE (R. B.). The etiology of infectious diarrhoea. (Winter scours) in cattle. J. Exp. Med., 1931, 53 : 835-843.

6. LESEL (R.). Etude d'un troupeau de bovins vivant sur l'Ile d'Amsterdam. Rev. Elev. Méd. vét. Pays trop., 1969, 22 (1): 107-125.

7. MacPHERSON (L. W.). Bovine virus enteritis
(Winter dysentery). Canad. J. Comp. Med., 1957, 21 (6) : 184-192.

8. Rapports annuels du Laboratoire et du Service de l'Elevage de Madagascar. Archives de l'L.E.M.V.T.

9. RENAULT (L.), VALLEE (A.), GAUTHIER (J.) et MAIRE (C.). Diagnostic de la colibacillose du veau. Rev. Méd. vét., 1968, 144 (4) : 315-322.

10. ROBERTS (S.J.). Winter dysentery in dairy cattle. Cornell Vet., 1957, 47: 372-388.

11. ROLLINDSON (D. H. L.). Infectious diarrhoea of dairy cows. Vet, Rec., 1948, 60 (17): 155-157.

12. SOJKA (W. J.). Escherichia coli in domestic animals and poultry. Farnham Royal, Bucks, Commonwealth Agricultural Bureaux, 1965. (C.A.B. Review Series no 7 ).

13. VAISSAIRE (J.), RENAULT (L.), MAIRE (C.), PALISSE (M.) et LINDER (T.). Contribution à l'étude de l'entérite hémorragique du porc. Rec. Méd. vét., 1970, 145 : 433-447. 